\title{
Malyugin's Theorems: A New Concept in Logical Control, VLSI Design, and Data Structures for New Technologies ${ }^{1}$
}

\author{
V. P. Shmerko \\ University of Calgary, Calgary, Canada \\ Strzelin Technical University, Strzelin, Poland \\ Received December 16, 2003
}

\begin{abstract}
Theoretical results due to V. Malyugin on linearization of arithmetical models of logical functions are interpreted and refined from the viewpoint of modern techniques of logical design of integral circuits and nearest neighbor technologies.
\end{abstract}

\section{INTRODUCTION}

Computer-aided design of integral circuits (CAD IC) consists in transforming the description of a circuit at the algorithmic or behaviorial level to a description at the physical level satisfying technological conditions. This process is subdivided into system, behavioral, logic and layout levels. This paper is concerned with the logic level, in which the behavior of a circuit is described by logical functions. The effectiveness of algorithms used in this level primarily depends on data structures (AND/OR forms, cubes, vector representations, etc.). Problems of large dimension are effectively solved with decision diagrams (DD) [1,2]. Arithmetical logic is the extension of the Boolean algebra through arithmetical operations permitting the representation of logical functions by integer functions (Boolean-to-integer functions). In many cases, this is helpful in manipulating and transforming data of CAD systems $[3,4]$.

Beginning from the pioneering works of Aiken on the application of arithmetical logic in designing the computers MARK 3 and MARK 4 (1952) [5], many papers have developed word-level structures, i.e., representation of data in the form of specially grouped words, to obtain compact data representation and thereby increase the dimension of problems, for example, by the so-called word-level $D D$. Another approach is developed in [6]. However, it is not clear which of the methods of formation of words from Boolean functions is preferable. The properties of arithmetical logic in this respect are rather attractive, for example, is it not possible to group data such that they are described by a linear arithmetical polynomial (LAR). In certain well-know publications, this problem is solved in a simplified formulation by partly linearizing the word-level model $[1,3,4,7]$. Fundamental results due to Malyugin $[8,9]$ pave the way for solving this problem.

This paper is devoted to linearization of arithmetical word-level models - an integral part of the Malyugin approach. Our aim is to interpret his approach to linearization of arithmetical models (polynomials) and refine it from the standpoint of new requirements and capabilities of modern CAD IC systems. In particular, we describe a classification for word-level models and show the importance of the cortege technique for linearization of arithmetical models. Jointly with linearization conditions and matrix transformation technique [10,11], the arithmetical model of a circuit (combination circuit or a circuit with memory on binary and multilevel gates) is shown to be linearizable [12]. Then, the linear model is directly mapped into a linear DD (LDD) having certain useful properties. For the first time we show that any circuit is representable by a system of $L D D$.

\footnotetext{
${ }^{1}$ This work was supported in part by the NATO Collaborative Linkage Grant PST/CLG 979071.
} 
Finally, using the properties of linear models, we solve the problem of large weight coefficients characteristic of arithmetical forms. It is a serious hurdle in practical application of arithmetical logic. Experiments with industrial circuits have shown that our algorithm is effective for solving large-dimensional problems (in experiments, coefficients took values up to $2^{100}$ ); none of the known methods can solve such problems. Latest results in arithmetical logic, including linearization of models, are reviewed in [13].

Arithmetic transformation methods are discussed in Sections 3-5. Linearization of arithmetic polynomials is described in Section 6. Section 7 is concerned with the computational aspects of linear transforms based on LAR. Section 8 is devoted to manipulations with large values of coefficients of LAR. Experimental results are given in Section 9. The application of arithmetic logic in prospective technologies is examined in Section 10.

\section{PRELIMINARIES}

\subsection{Word-level Forms}

A Boolean function of $n$ variables is a mapping $\{0,1\}^{n} \rightarrow\{0,1\}$. The word-level form in arithmetic logic is an integer function $P$ with a mapping $\{0,1\}^{n} \rightarrow\{0,1, \ldots, p-1\}$, where $p>2$. In general, a system of $r$ Boolean functions of $n$ variables ( $r$-output Boolean function) can be represented by an integer function $D$ with the mapping $\{0,1\}^{n} \rightarrow\left\{0,1, \ldots, 2^{r}-1\right\}$. Word-level representation of a system of Boolean functions is defined to be an ordering of integer functions $P_{j}$ such that the functions form a word

$$
D=2^{r-1} P_{r}+\ldots+2^{1} P_{2}+2^{0} P_{1} .
$$

The outputs $f_{1}, f_{2}, \ldots, f_{r}$ of a Boolean function $f$ are uniquely estimated from expression (1) through transformation of $P_{j}$ into $f_{j}$. This form is the weighted sum of $r$ arithmetic polynomials $P_{j}$, $j=1, \ldots, r$. A particular case of this expression is the linear arithmetic polynomial (LAR), which contains not more than $(n+1)$ nonzero coefficients. Transformation of an arithmetic polynomial (1) to a LAR is the solution of the boundary-value problem of arithmetic logic. In this paper, we focus our attention on methods of formation of LAR and their properties.

In the general case, a word-level model is described by a sequence of words. A word contains information on a function and components of a system (circuit). The simplest word-level model contains only Boolean constants. For example, the word 101 represents a binary word. In CAD IC, we use different types of word-level models, for instance, sum of weighted Boolean expressions or arithmetic polynomials (1). Word-level structures are manipulated and transformed by a special technique based on decision diagrams, known as the WDD [2]. The initial word-level description may contain arithmetical expressions or truth vectors of Boolean functions. Malyugin refers to such data structures as corteges and has developed a technique for manipulating over them [8].

\subsection{Linearization Problem}

Arithmetic logic is an extension of Boolean algebra. An arithmetical expression is formed via replacement of Boolean operations by arithmetical operations over integer data according to certain rules. Algebra of corteges is an extension of arithmetical logic. ${ }^{2}$

${ }^{2}$ An extension of a field $K$ is obtained by including new elements $r_{1}, r_{2}, \ldots, r_{n}$ such that $F=K\left(r_{1}, r_{2}, \ldots, r_{n}\right)$, where $r_{1}, r_{2}, \ldots, r_{n} \in F$. The resultant field $F$ is called the extension of the field $K$ if all group operations and field properties are preserved. 
Definition 1. The LAR of a Boolean function $f$ of $n$ variables $x_{1}, \ldots, x_{n}$ is

$$
D=d_{0}+\sum_{i=1}^{n} d_{i} x_{i}=d_{0}+d_{1} x_{1}+\ldots+d_{n} x_{n},
$$

where $d_{i}(i=1,2, \ldots, n)$ is the $i$ th integer coefficient.

According to expression (2), an $r$-output Boolean function can be represented by a LAR if all polynomials $P_{j}$ in (1) are LARs (trivial case) or (b) the weighted sum of (1) contains LARs and nonlinear polynomials $P_{j}$ (the general case).

Linearization means transformation of a nonlinear arithmetic polynomial (1) into a linear arithmetic polynomial (2) with not more than $(n+1)$ nonzero coefficients. Probably, a similar linearization problem was first solved in [16], though general interest in this topic had emerged as early as 1952. (see [17]). A solution in the spectral domain is given in $[18,19]$.

Example 1. The outputs $f_{1}=x_{1} \oplus x_{2}$ and $f_{2}=x_{1} x_{2}$ of a half-adder are nonlinear arithmetic polynomials $P_{1}=x_{1}+x_{2}-2 x_{1} x_{2}$ and $P_{2}=x_{1} x_{2}$, respectively. This is the general case of design of LARs (2). According to (1), $D=2^{1} P_{2}+2^{0} P_{1}=2^{1} x_{1} x_{2}+2^{0}\left(x_{1}+x_{2}-2 x_{1} x_{2}\right)=x_{1}+x_{2}$ is a LAR. Interchanging the numbers of $P_{1}$ and $P_{2}$, we obtain a nonlinear polynomial $D=2 x_{1}+2 x_{2}-3 x_{1} x_{2}$.

In this example, identical nonlinear terms $2 x_{1} x_{2}$ have been eliminated as a result of their unlike signs by a suitable choice of order of numeration for the outputs of the Boolean function. Consequently, linearization of expression (1) is sensitive to the order of numeration of outputs of the Boolean function in the word-level representation. If this order is suitably chosen, then the LAR uniquely represents a function, and this provides a method for estimating the initial function.

The linearization problem for data structures can also be formulated differently: find a linear transformation $L$ for the variables $x_{1}, x_{2}, \ldots, x_{n}$ of a Boolean function $f$ such that $f=L\{\widetilde{f}\}$. If the Boolean function $\widetilde{f}$ is simpler to compute than the function $f$ and the transformation $L$ does not require sophisticated computation resources, then this approach is useful in designing and analyzing logical circuits $[7,20,21]$.

\subsection{Topic of Study}

The state-of-the-art of our problem shows that there are two main hurdles in the way of practical application of arithmetical logic to logical design and control, viz.:

(i) Absence of satisfactory methods for linearization of arithmetical models. Although the results due to Malyugin give a "key" to many real problems, his linearization approach needs refinement from the viewpoint of modern CAD IC technology.

(ii) Impossibility of utilization of the existing methods for solving large-dimensional problems. Description of data structures by arithmetical polynomials and manipulations over them requires huge weight coefficients even for small circuits. If this hurdle is surmounted, vistas open for the utilization of the word-level technique, in particular, WDD technology.

Motivated by these facts, we shall focus our attention on linear models of arithmetical logic for multilevel circuits and for transforming them to an LDD set:

$$
\text { Function (circuit) } \Longleftrightarrow \text { LAR } \Longleftrightarrow \text { LDD } \Longleftrightarrow \text { Realization. }
$$

Thus we can attain the main goal: application of Malyugin's results in CAD IC, their interpretation in terms of modern word-level technology, and solution of certain related problems, such as transmission of data via LDD networks and manipulations with large weight coefficients. 


\begin{tabular}{|c|c|c|c|}
\hline $\begin{array}{l}\text { An arithmetical } \\
\text { polynomial } \\
\text { to algebraic form } \\
P_{i}\left(x_{1}, x_{2}, \ldots, x_{n}\right)\end{array}$ & $\begin{array}{c}\text { An arithmetical } \\
\text { polynomial } \\
\text { to vector form } \\
{\left[P_{r}, \ldots, P_{2} P_{1}\right]}\end{array}$ & $\begin{array}{l}\text { An arithmetical } \\
\text { polynomial } \\
\text { to algebraic form } \\
P_{i}\left(x_{1}, x_{2}, \ldots, x_{n}\right)\end{array}$ & $\begin{array}{l}\text { An arithmetical } \\
\text { polynomial } \\
\text { to algebraic form } \\
P_{i}\left(x_{1}, x_{2}, \ldots, x_{n}\right)\end{array}$ \\
\hline$\sqrt{\square}$ & 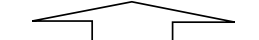 & S & $\rightarrow$ \\
\hline $\begin{array}{l}\text { A Boolean function } \\
\text { to algebraic form } \\
f_{i}\left(x_{1}, x_{2}, \ldots, x_{n}\right)\end{array}$ & $\begin{array}{c}\text { A Boolean function } \\
\text { to vector form } \\
{\left[X_{r} \ldots X_{2} X_{1}\right]}\end{array}$ & $\begin{array}{l}\text { A Boolean function } \\
\text { to Taylor kernel } \\
\text { form }\end{array}$ & $\begin{array}{l}\text { A Boolean function } \\
\text { to cortege form } \\
f_{r} \odot \ldots \odot f_{2} \odot f_{1}\end{array}$ \\
\hline
\end{tabular}

Fig. 1. Four methods of transformation of a Boolean function to an arithmetical polynomial (left to right): algebraic, matrix, Taylor expansion, via algebra of corteges (Malyugin's method).

To determine the place and role of Malyugin's linearization methods in modern word-level technology, we must classify methods of realization of arithmetical transformations, by which we mean the relationship between Boolean functions and their representation by arithmetical polynomials. ${ }^{3}$

Direct arithmetical transformation is used for solving the following problem: given a Boolean function (a many-output function in the general case), find its corresponding arithmetical polynomial. Inverse arithmetical transformation is used for estimating the Boolean function from a given polynomial. Consequently, arithmetical transformation is defined by a pair of relations for solving direct and inverse problems.

In this paper, we use four methods for manipulations over arithmetical expressions: algebraic, matrix, Taylor expansion, and algebra of corteges based on the Malyugin method (Fig. 1). ${ }^{4}$

Algebraic manipulations based on the linearity of arithmetical transformations. They feature clarity and simplicity, and helpful in solving large-dimensional problems. For details, see Section 3.

Matrix manipulations are based on the transformations used in digital signal processing. They are suitable for solving large-dimensional problems in combination with graphic structure of data, such as DD. Details are presented in Section 4.

Taylor expansion is the analog of the Taylor series in mathematical analysis. Its merits are that the coefficients of an arithmetical polynomial admit representation in a convenient form for analysis by analogs of Boolean differences (as arithmetical derivatives).

Manipulations based on the algebra of corteges - the topic for discussion and investigation in this paper-underlie the Malyugin approach, which significantly differs from the first three approaches. Its merits are $(i)$ algorithmically flexible manipulation over large-dimensional arithmetical expressions, (ii) new possibilities for realization of direct and inverse transformations, (iii) simplicity of design and analysis of LARs, and (vi) possibility of representation of any Boolean function by a finite LAR set. The last property is not inherent in any of the well-known method. The Malyugin method is described in Section 5.

These transformation methods differ in computational complexity and algorithmic realizability. The choice of a method depends on the concrete problem.

\footnotetext{
${ }^{3}$ In scientific literature, arithmetical transformation is often interpreted in terms of signal theory: a Boolean function is a representation of a signal in time domain and its corresponding arithmetical polynomial is a form of representation of signals in the spectral domain $[15,22]$.

${ }^{4}$ This classification does not include the well-known methods of approximate computation of coefficients of arithmetical polynomials via probabilistic transformations [14, 15].
} 


\section{TRANSFORMATION TO ALGEBRAIC FORM}

Arithmetical transformation can be realized directly by representing every element in a logical circuit by an appropriate polynomial [23]. This is one of merits of the algebraic form.

\subsection{Direct Transformation}

Manipulations over arithmetical expressions are based on the linearity of arithmetical transformations. Let $\mathcal{A}\{f\}$ denote the arithmetical transform of a Boolean function $f$ represented by $f=f_{1} \vee f_{2} \ldots \vee f_{r}$. Then the arithmetical analog of the Boolean function $\mathcal{A}\{f\}$ is constructed via transformation of subfunctions $f_{i}$

$$
\mathcal{A}\{f\}=\mathcal{A}\left\{f_{1} \vee f_{2} \ldots \vee f_{r}\right\}=\mathcal{A}\left\{f_{1}\right\}+\mathcal{A}\left\{f_{2}\right\} \ldots+\mathcal{A}\left\{f_{r}\right\} .
$$

Example 2. Using the linearity property, the trivial equalities $\bar{x}=1-x, x_{1} \vee x_{2}=x_{1}+x_{2}-x_{1} x_{2}$, $x_{1} x_{2}=x_{1} x_{2}$, and $x_{1} \oplus x_{2}=x_{1}+x_{2}-2 x_{1} x_{2}$ can be generalized to functions. The Boolean functions $f_{1}=x_{1} \bar{x}_{2} \vee x_{1} \bar{x}_{3}, f_{2}=x_{1} \vee\left(x_{2} \oplus x_{3}\right)$ and $f_{3}=x_{1} \oplus x_{2}$ are reduced to arithmetical form as

$$
\begin{gathered}
\mathcal{A}\left\{f_{1}\right\}=\mathcal{A}\left\{x_{1} \bar{x}_{2}\right\}+\mathcal{A}\left\{x_{1} \bar{x}_{3}\right\}-\mathcal{A}\left\{x_{1} \bar{x}_{2}\right\} \mathcal{A}\left\{x_{1} \bar{x}_{3}\right\}=x_{1} \bar{x}_{2}+x_{1} \bar{x}_{3}-x_{1} \bar{x}_{2} \bar{x}_{3}, \\
\mathcal{A}\left\{f_{2}\right\}=x_{1}+x_{2}+x_{3}-x_{1} x_{2}-x_{1} x_{3}-2 x_{2} x_{3}+2 x_{1} x_{2} x_{3}, \\
\mathcal{A}\left\{f_{3}\right\}=x_{1}-2 x_{1} x_{2}+x_{2} .
\end{gathered}
$$

Since arithmetical transforms are linear, the outputs of the Boolean function $f$ can be grouped as words (1), i.e., functions of every output can be represented by the weighted sum of transforms

$$
\mathcal{A}\{f\}=\mathcal{A}\left\{2^{r-1} f_{r}\right\}+\ldots+\mathcal{A}\left\{2^{1} f_{2}\right\}+\mathcal{A}\left\{2^{0} f_{1}\right\}=2^{r-1} P_{r}+\ldots+2^{1} P_{2}+2^{0} P_{1}=D .
$$

Example 3. Applying property (3) to the Boolean function in Example 2, we obtain

$$
D=2^{0} f_{1}+2^{1} f_{2}+2^{2} f_{3}=7 x_{1}+6 x_{2}+2 x_{3}-10 x_{1} x_{2}-4 x_{1} x_{3}-4 x_{2} x_{3}+3 x_{1} x_{2} x_{3} .
$$

In this example, three Boolean functions, each of which is represented by an arithmetical equivalent, are described by one (nonlinear) polynomial, i.e, in word-level form. This polynomial is obviously inferior in complexity to the initial Boolean functions. All methods of grouping of functions (numeration of outputs of the function as in Example 1) do not yield the linear expression (2). This is a motivation for developing new linearization methods.

\subsection{Inverse Transformation}

Since inverse transformation to algebraic form mostly requires an intuitive approach and hardly yields to algorithmization, it is not of much interest even for small-dimensional problems.

\section{TRANSFORMATION TO MATRIX FORM}

Here we examine two matrix arithmetical transformations [11]. Let $\mathbf{X}$ and $\mathbf{D}$ be the truth vector of a Boolean function $f$ and the vector of coefficients of its arithmetical representation, respectively. The relationship between $\mathbf{X}$ and $\mathbf{P}$ is defined by two transformations

$$
\begin{gathered}
\mathbf{D}=\mathbf{A}_{2^{n}} \mathbf{X} \text { (direct transformation), } \\
\mathbf{X}=\mathbf{A}_{2^{n}}^{-1} \mathbf{D} \text { (inverse transformation), }
\end{gathered}
$$

where $\mathbf{A}_{2^{n}}$ and $\mathbf{A}_{2^{n}}^{-1}$ are the matrices of direct and inverse arithmetical transformations, respectively. 


\subsection{Direct Transformation}

Direct transformation (4) is applied to represent a Boolean function in arithmetical form defined by the coefficient vector $\mathbf{D} .{ }^{5}$ The arithmetical transformation matrix $A_{2^{n}}$ is formed by the rule

$$
\mathbf{A}_{2^{n}}=\bigotimes_{i=1}^{n} \mathbf{A}_{1}, \quad \mathbf{A}_{1}=\left[\begin{array}{rr}
1 & 0 \\
-1 & 1
\end{array}\right]
$$

where $A_{1}$ is the base matrix of direct transformation and $\otimes$ denotes the Kronecker multiplication.

Example 4. The three-output Boolean function of Example 3 is transformed to an arithmetical polynomial as follows. First we form its truth vector with integral elements

$$
\mathbf{X}=\underbrace{\left[\mathbf{X}_{3}\left|\mathbf{X}_{2}\right| \mathbf{X}_{1}\right]}_{\text {Truth matrix }}=\left[\begin{array}{ccc}
0 & 0 & 0 \\
0 & 1 & 0 \\
1 & 1 & 0 \\
1 & 0 & 0 \\
1 & 1 & 1 \\
1 & 0 & 1 \\
0 & 0 & 1 \\
0 & 1 & 0
\end{array}\right]=\left[\begin{array}{l}
0 \\
2 \\
6 \\
4 \\
7 \\
5 \\
1 \\
2
\end{array}\right]
$$

Then applying the direct transformation (4), we obtain the coefficient vector $\mathbf{D}$ of the unknown arithmetical polynomial $D$

$$
\mathbf{D}=\mathbf{A}_{2^{3}} \mathbf{X}=\left[\begin{array}{rrrrrrrr}
1 & 0 & 0 & 0 & 0 & 0 & 0 & 0 \\
-1 & 1 & 0 & 0 & 0 & 0 & 0 & 0 \\
-1 & 0 & 1 & 0 & 0 & 0 & 0 & 0 \\
1 & -1 & -1 & 1 & 0 & 0 & 0 & 0 \\
-1 & 0 & 0 & 0 & 1 & 0 & 0 & 0 \\
1 & -1 & 0 & 0 & -1 & 1 & 0 & 0 \\
1 & 0 & -1 & 0 & -1 & 0 & 1 & 0 \\
-1 & 1 & 1 & -1 & 1 & -1 & -1 & 1
\end{array}\right]\left[\begin{array}{l}
0 \\
2 \\
6 \\
4 \\
7 \\
5 \\
1 \\
2
\end{array}\right]=\left[\begin{array}{l}
0 \\
2 \\
6 \\
4 \\
7 \\
5 \\
1 \\
2
\end{array}\right] \begin{aligned}
& x_{3} \\
& x_{2} \\
& x_{2} x_{3} \\
& x_{1} \\
& x_{1} x_{3} \\
& x_{1} x_{2} \\
& x_{1} x_{2} x_{3}
\end{aligned}
$$

This example shows that matrix form is preferable to algebraic form in computations.

\subsection{Inverse Transformation}

Inverse transformation applied to the coefficient vector $D$ (4) yields the truth vector $\mathbf{X}$. The matrix of inverse arithmetical transformation $A^{-1}(n)$ is formed according to the rule

$$
\mathbf{A}_{2^{n}}^{-1}=\bigotimes_{i=1}^{n} \mathbf{A}_{1}^{-1}, \quad \mathbf{A}_{1}^{-1}=\left[\begin{array}{ll}
1 & 0 \\
1 & 1
\end{array}\right],
$$

where $A_{1}^{-1}$ is the base matrix of inverse transformation.

Example 5. Find the Boolean function corresponding to $L A R=2+3 x_{1}+5 x_{2}$. The inverse transformation (5) generates the truth vector $\mathbf{X}$

$$
\mathbf{X}=\mathbf{A}_{2^{n}}^{-1} \mathbf{D}=\left[\begin{array}{llll}
1 & 0 & 0 & 0 \\
1 & 1 & 0 & 0 \\
1 & 0 & 1 & 0 \\
1 & 1 & 1 & 1
\end{array}\right]\left[\begin{array}{l}
2 \\
5 \\
3 \\
0
\end{array}\right]=\left[\begin{array}{r}
2 \\
7 \\
5 \\
10
\end{array}\right]=\left[\begin{array}{llll}
0 & 0 & 1 & 0 \\
0 & 1 & 1 & 1 \\
0 & 1 & 0 & 1 \\
1 & 0 & 1 & 0
\end{array}\right]
$$

Consequently, $L A R=2+3 x_{1}+5 x_{2}$ represents a Boolean function of four outputs $f_{4}=x_{1} x_{2}$, $f_{3}=x_{1} \oplus x_{2}, f_{2}=\bar{x}_{1} \oplus x_{1} x_{2}$, and $f_{1}=x_{1} \oplus x_{2}$.

\footnotetext{
${ }^{5}$ The coefficient vector is also called the arithmetical spectrum of the Boolean function.
} 


\section{TRANSFORMATIONS IN CORTEGE ALGEBRA}

A common drawback of these three methods of arithmetical transformations (Fig. 1) is the restricted possibilities for manipulations over functions in word-level representation for linearization. Widening these possibilities, Malyugin solved the following problem: given a many-output Boolean function, find (a) an algebra for effective manipulation over word-level structured data and (b) a method for linearization of arithmetical expressions in this algebra.

\subsection{Initial Definitions}

Without specifying the nature of an object (element), a cortege $\mathrm{Cor}_{m}$ is defined to be an ordered sequence of $m$ elements $U_{i}, i=1,2, \ldots, m$ :

$$
U_{m} \odot U_{m-1} \odot \cdots \odot U_{1}=\operatorname{Cor}_{m}=\bigodot_{i=1}^{m} U_{i},
$$

where $\odot$ denotes the separator between elements. Often we write ${ }^{A} \operatorname{Cor}_{m}$ to denote that the cortege $A$ contains $m$ elements. In arithmetical logic, elements of a cortege may be constants 0 and 1 , variables $x_{i}$, functions $f_{i}$ (Boolean expressions), and (nonlinear) polynomials (in the general case), denoted in the sequel by $A R_{i}$.

Example 6. (i) $C o r=0 \odot x_{3} \odot 1$ is a cortege of constants, (ii) Cor $=f_{3} \odot f_{2} \odot f_{1}$ is a cortege of functions, (iii) $C o r=A R_{3} \odot A R_{2} \odot A R_{1}$ is a cortege of polynomials, and (iv) Cor $=\left(x_{2} \vee x_{3}\right) \odot$ $\left(x_{1} \oplus x_{2}\right) \odot 0$ is a cortege of Boolean expressions and a constant.

The number of an element in a cortege is its address. The advantage of description by a cortege is the possibility for grouping and manipulation over data in word-level format. For example, $L A R=x_{1}+x_{2}$ represents a Boolean function with two outputs $x_{1} x_{2}$ and $x_{1} \oplus x_{2}$, which is defined by the cortege $x_{1}+x_{2} \Longleftrightarrow \underbrace{\left(x_{1} x_{2}\right)}_{\text {Higher-order bit }} \odot \underbrace{\left(x_{1} \oplus x_{2}\right)}_{\text {Lower-order bit }}$. The masking operator is designed to extract the desired function from a cortege or LAR.

Definition 2. The masking operator

$$
f_{r}=\Xi_{r}^{m}\{f\}
$$

shows the position $r$ of the function $f_{r}$ in the cortege $\operatorname{Cor}_{m}$ of $m$ functions $f_{i}, i=1,2, \ldots, m$.

Example 7. Functions in a cortege $x_{1} x_{2} \odot\left(x_{1} \oplus x_{2}\right)$ are "extracted" with a masking operator $x_{1} x_{2}=\boldsymbol{\Xi}_{2}^{2}\left\{x_{1}+x_{2}\right\}$ and $x_{1} \oplus x_{2}=\boldsymbol{\Xi}_{2}^{1}\left\{x_{1}+x_{2}\right\}$.

\subsection{Cortege Algebra}

The main requirements imposed on the algebra used in the description and transformation of switching functions are functional completeness (distinguishable algebraic representation for a function), flexibility (reasonable complexity of data manipulation algorithms), and realizability (simplicity and obvious physical interpretation of the relationship with other data structures). Using these requirements, Malyugin extended the classical Boolean algebra to operations over corteges.

\subsection{Sum of Corteges}

We can find the sum of a cortege $A$ of elements $a_{i}$ and a cortege $B$ of elements $b_{i}$ in analogy with the design of a binary adder, whose primitive components are called half-adders. ${ }^{6}$ Let us

${ }^{6}$ A binary half-adder computes the sum of two binary numbers: $0+0=0,0+1=1,1+0=1$, and $1+1=10$ (Fig. 2). The results of the first three operations are represented by only one bit. But if both operands are 1 , the sum is represented by two bits, which correspond to CARRY and SUM. 
(a)

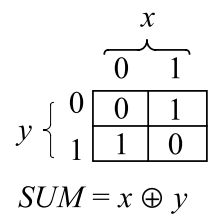

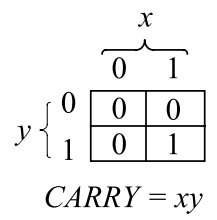

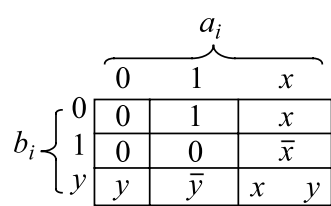

$S U M=a_{i} \oplus b_{i}$ (b)

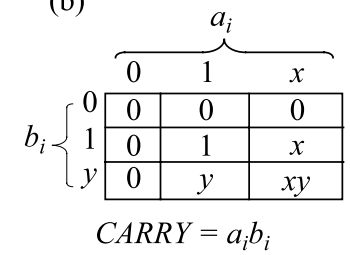

Fig. 2. Truth tables of a binary half-adder (a) and cortege half-adder (b) (8).

\begin{tabular}{|c|c|c|c|c|c|c|c|c|c|}
\hline \multicolumn{10}{|c|}{00} \\
\hline 0 & 0 & 1 & $x$ & 1 & 0 & $\bar{x}$ & $x$ & $\bar{x}$ & 0 \\
\hline 1 & 1 & 0 & $\bar{x}$ & 0 & 1 & $x$ & $\bar{x}$ & $x$ & 1 \\
\hline$y$ & $y$ & $\bar{y}$ & $x \oplus y$ & $\bar{y}$ & $y$ & $\bar{x} \oplus y$ & $x \oplus y$ & $\bar{x} \oplus y$ & $y$ \\
\hline$a$ & $\mathrm{Cal}$ & & & & & & & & \\
\hline$b_{i}$ & 00 & 01 & $0 x$ & 10 & 11 & $1 x$ & $x 0$ & $x 1$ & $x x$ \\
\hline 0 & 0 & 0 & 0 & 0 & 1 & $x$ & 0 & $x$ & $x$ \\
\hline 1 & 0 & 1 & $x y$ & $y$ & 1 & $x \vee y$ & $x y$ & $x \vee y$ & $x$ \\
\hline$y$ & 0 & $y$ & $x y$ & $y$ & $y$ & $x \vee y$ & $x y$ & $x \vee y$ & $x$ \\
\hline
\end{tabular}

Fig. 3. Truth tables of a complete two-input cortege adder (9) (top table for Sum and bottom table for Carry).

design a half-adder for corteges. Since the elements of a cortege may be constants 0 , 1 , variables, or Boolean expressions, let us express the initial data as $a_{i}, b_{i} \in\{0,1, x, y\}$, where $x$ and $y$ are Boolean variables. Addition of constants 0 and 1 differs from the functions of a binary half-adder only in the form of expressions $0+0=0 \odot 0,0+1=0 \odot 1,1+0=0 \odot 1$, and $1+1=1 \odot 0$. For the addition of constants and variables, there are four situations $(a) 0+x=0 \odot x,(b) 1+x=x \odot \bar{x}$, (c) $y+0=0 \odot y$, and $(d) y+1=1 \odot \bar{y}$, since carry operation in the addition of $(1+x)$ and $(1+y)$ is formed only for $x=y=1$. Finally, $x+y=(x \oplus y) \odot x y$, because $S U M=x \oplus y$ and $C A R R Y=x y$ in the binary half-adder. From the truth tables for a half-adder of corteges and a binary half-adder shown in Fig. 2 we arrive at

Assertion 1. Binary half-adders and cortege half-adders have identical formal descriptions

$$
S U M=a_{i} \oplus b_{i}, \quad C A R R Y=a_{i} b_{i} .
$$

Using half-adder (8), we can design a complete adder of corteges. ${ }^{7}$ Addition of constants is implemented as in a binary adder, including the case $1+1+1=1 \odot 1$. The four cases considered above for the addition of constants 0 and 1 and variables $x$ and $y$ are similar to those for a cortege half-adder. The result of addition for other combinations of constants and variables is (i) $1+x+1=1 \odot x,(i i) 1+y+1=1 \odot y$, and $($ iii $) x+1+y=0 \odot(\bar{x} \oplus y)$ since $x+1+y=$ $(x+1)+y=(x \odot \bar{x})+y=(x \oplus \bar{x} y) \odot(\bar{x} \oplus y)=(x \vee y) \odot(\bar{x} \oplus y)$. Finally, the sum of three elements of a cortege for $x+x+y$ and $x+y+y$ is $x+x+y=(x+x)+y=(x \odot 0)+y=x \odot y$. From the truth tables of adders for the addition of binary numbers and corteges (Fig. 3), we arrive at

Assertion 2. Formal descriptions of binary adders and cortege adders are identical

$$
S U M=a_{i} \oplus b_{i} \oplus C A R R Y_{i-1}, \quad C A R R Y_{i}=a_{i} b_{i} \vee C A R R Y_{i-1}\left(a_{i} \oplus b_{i}\right) .
$$

\footnotetext{
$\overline{7}$ A two-bit adder has two inputs for terms $x$ and $y$ and an input for carrying from the preceding digit $C A R R Y_{i-1}$. The formal model is defined by two relations $S U M=x \oplus y \oplus C A R R Y_{i-1}$ and $C A R R Y=x y+C A R R Y_{i-1}(x \oplus y)$.
} 


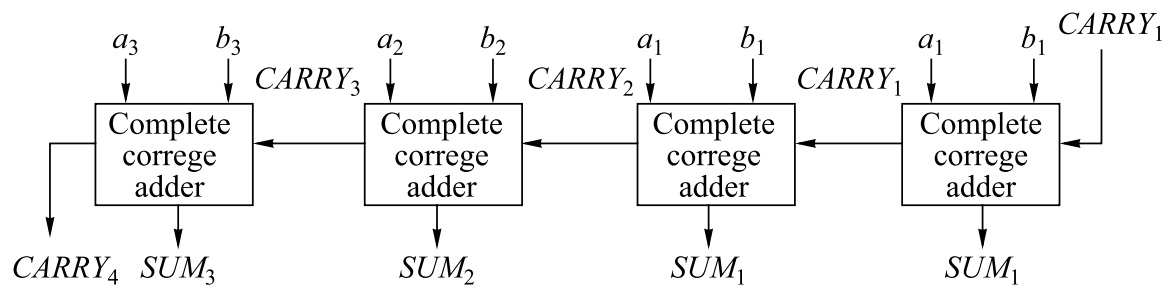

Fig. 4. A sequential adder of four-element corteges.

Interpretation of addition of corteges by an adder (9) is useful in designing algorithms for manipulations over corteges. For example, the analog of a parallel adder of corteges is the parallel binary adder, and the algorithm for sequential addition of corteges is derived from the architecture of the sequential binary adder (Fig. 4).

\subsection{Product of Corteges}

The product of two corteges is conveniently interpreted with a binary multiplier based on the accumulation of partial products. The product of two corteges ${ }^{A} \mathrm{Cor}_{2}=x \odot 1$ and ${ }^{B} \mathrm{Cor}_{2}=0 \odot y$ is

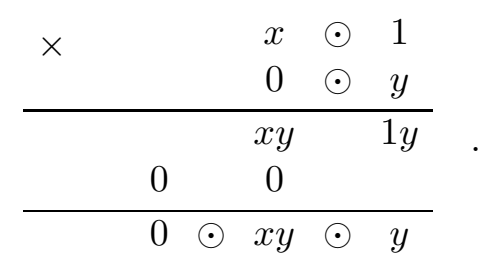

Here AND-terms are partial products. The result ${ }^{A} \mathrm{Cor}_{2} \times{ }^{B} \mathrm{Cor}_{2}=0 \odot x y \odot y$ is formed by accumulating partial products, carrying digits from right to left.

\subsection{Properties of Cortege Algebra}

Let three corteges ${ }^{A} \mathrm{Cor}_{m},{ }^{B} \mathrm{Cor}_{s}$, and ${ }^{C} \mathrm{Cor}_{k}$ be given. To extend the Malyugin arithmetical logic, let us define a few axioms of cortege algebra ( 0 and 1 elements, complementation, commutativity, associativity, and distributivity). Using them, we can implement any cortege transformation.

Example 8. A cortege $\mathrm{Cor}_{k}$ is given. A cortege with inverse order of occurrence of elements is formed as follows. Since $\operatorname{Cor}_{k}+\overline{\operatorname{Cor}}_{k}=2^{k}-1$, we have $\overline{\operatorname{Cor}}_{k}=2^{k}-1-\operatorname{Cor}_{k}$. The inverse of the cortege $\operatorname{Cor}_{2}=x_{1} \odot x_{1} x_{2}$ is the cortege $\overline{\operatorname{Cor}}_{2}=2^{2}-1-\operatorname{Cor}_{2}=3-x_{1} \odot x_{1} x_{2}=(1 \odot 1)-\left(x_{1} \odot x_{1} x_{2}\right)=$ $1-x_{1} \odot 1-x_{1} x_{2}=\bar{x}_{1} \odot \bar{x}_{1} x_{2}$, where $3=1 \odot 1$, or in vector form

$$
\mathrm{Cor}_{2}=x_{1} \odot x_{1} x_{2}=\left[\begin{array}{l}
0 \\
0 \\
1 \\
1
\end{array}\right] \odot\left[\begin{array}{l}
0 \\
0 \\
0 \\
1
\end{array}\right], \quad \overline{\operatorname{Cor}}_{2}=\bar{x}_{1} \odot{\overline{x_{1} x_{2}}}=\left[\begin{array}{l}
1 \\
1 \\
0 \\
0
\end{array}\right] \odot\left[\begin{array}{l}
1 \\
1 \\
1 \\
0
\end{array}\right] .
$$

\subsection{Particular Cases}

Particular cases of cortege operations are helpful in simplifying transformations and designing algorithms.

Addition of a constant and a function. Let us express the relation $x_{1}+x_{2}=x_{1} x_{2} \odot\left(x_{1} \oplus x_{2}\right)$ between a function $f$ and a constant $a \in\{0,1\}$ as

$$
a+f=a f \odot(a \oplus f) .
$$


Relation (10) cannot be used for manipulations over corteges since it holds only for single-bit operations. For $a=0$, we have $0+f=0 f \odot(0 \oplus f)=f$. Similarly, for $a=1$ we obtain $1+f=1 f \odot(1 \oplus f)=f \odot \bar{f}$. Let us find $a+f$ for $a>1$. Let $a=2$. Since $a=2$ and the function $f$ is represented as a cortege of $1 \odot 0$ and $f=0 \odot f$, we have

$$
\begin{aligned}
2 & =1 \odot 0 \\
f & =0 \odot f \\
\hline 2+f & =1 \odot f
\end{aligned} .
$$

Multiplication of a constant by a function. In analogy with addition, for $a=3=1 \odot 1$ we obtain $3+\overline{f=f \odot \bar{f} \odot \bar{f} \text {. Since } 0 f=f, 1 f=f \text {, and }} 2=1 \odot 0$, the product of 2 and $f$ is

$$
\begin{aligned}
\wedge 2 & =1 \odot 0 \\
f & =
\end{aligned}
$$

Technique of manipulations over corteges. Example 9. The sum and product of a function $f=$

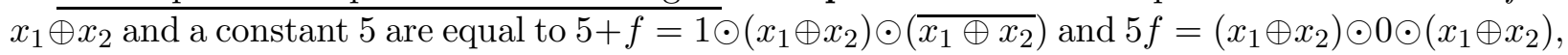
respectively, or in vector form

$$
5+f=5+\left[\begin{array}{l}
0 \\
1 \\
1 \\
0
\end{array}\right]=\left[\begin{array}{l}
1 \\
1 \\
1 \\
1
\end{array}\right] \odot\left[\begin{array}{l}
0 \\
1 \\
1 \\
0
\end{array}\right] \odot\left[\begin{array}{l}
1 \\
0 \\
0 \\
1
\end{array}\right], \quad 5 f=5\left[\begin{array}{l}
0 \\
1 \\
1 \\
0
\end{array}\right]=\left[\begin{array}{l}
0 \\
1 \\
1 \\
0
\end{array}\right] \odot\left[\begin{array}{l}
0 \\
0 \\
0 \\
0
\end{array}\right] \odot\left[\begin{array}{l}
0 \\
1 \\
1 \\
0
\end{array}\right]
$$

Since $x_{1} \oplus x_{2}=x_{1}+x_{2}-2 x_{1} x_{2}$, the result in arithmetical polynomial form is $5+f=5+x_{1}+$ $x_{2}-2 x_{1} x_{2}, 5 f=5\left(x_{1}+x_{2}-2 x_{1} x_{2}\right)$.

The next example illustrates the technique of manipulations involved in the addition of a cortege Cor and a constant $a$, i.e., $C o r+a$.

Example 10. Given a cortege $C o r=\left(x_{1} \vee x_{2}\right) \odot x_{2}$ and a constant $a=1$, find a new cortege Cor $=\left\{\left(x_{1} \vee x_{2}\right) \odot x_{2}\right\}+1$. Let us apply the computation scheme

$$
\begin{array}{clllll}
+ \text { Cor } & = & x_{1} \vee x_{2} & \odot & x_{2} \\
1 & = & 0 & \odot & 1 \\
\hline C o r+1 & = & x_{2} \odot & x_{1} \bar{x}_{2} & \odot & \bar{x}_{2}
\end{array} .
$$

For the lower-order digit, we have $x_{2}+1=x_{2} \odot \bar{x}_{2}$. The next digit is $\left(x_{1} \vee x_{2}\right)+0+x_{2}=\left(x_{1} \vee x_{2}\right)+x_{2}$. Using the relation $f_{1}+f_{2}=f_{1} f_{2} \odot\left(f_{1} \oplus f_{2}\right)$, we obtain $\left(x_{1} \vee x_{2}\right) x_{2} \odot\left(x_{1} \vee x_{2}\right) \oplus x_{2}=x_{2} \odot x_{1} \bar{x}_{2}$. The same result is obtained by computation in vector form

$$
1+\operatorname{Cor}=1+\left[\begin{array}{l}
0 \\
1 \\
1 \\
1
\end{array}\right] \odot\left[\begin{array}{l}
0 \\
1 \\
0 \\
1
\end{array}\right]=\left[\begin{array}{l}
0 \\
1 \\
0 \\
1
\end{array}\right] \odot\left[\begin{array}{l}
0 \\
0 \\
1 \\
0
\end{array}\right] \odot\left[\begin{array}{l}
1 \\
0 \\
1 \\
0
\end{array}\right]=x_{2} \odot x_{1} \bar{x}_{2} \odot \bar{x}_{2}
$$

The result of Example 10 is interpreted in terms of arithmetical logic as follows: the initial polynomial $A R=2\left(x_{1} \vee x_{2}\right)+x_{2}=2 x_{1}+3 x_{2}-2 x_{1} x_{2}$ is transformed to the polynomial $A R+1=$ $2^{2} x_{2}+2^{1} x_{1} \bar{x}_{2}+2^{0} \bar{x}_{2}=4 x_{2}+2 x_{1} \bar{x}_{2}+\bar{x}_{2}$.

\subsection{Direct and Inverse Transformations}

Direct arithmetical transformation is helpful in representing any Boolean function as an arithmetical polynomial with the help of cortege algebra. The initial function can be defined by Boolean expressions, a cortege, or a truth table. 
Given an arithmetical polynomial $A R$, find its corresponding cortege Cor of Boolean functions, i.e., $\mathcal{A}^{-1}(A R)=$ Cor . The solution algorithm consists of (a) representation of every term of the polynomial $A R$ by a cortege (in the general case, the polynomial may contain $2^{n}$ terms, i.e., $2^{n}$ corteges), (b) specification of the general format $m$ for all corteges, and (c) computation of the sum of corteges. Formally, the solution is guaranteed by

Theorem 1 ([9]). A cortege of Boolean functions is uniquely estimated from a given arithmetical polynomial $A R$ by the rule

$$
\mathcal{A}^{-1}(A R)=\operatorname{Cor}_{m}=\sum_{i=0}^{2^{n}-1} \bigodot_{j=1}^{m} \tau_{i j},
$$

where $\tau_{i j}$ is the $j$ th element in the ith cortege (term of the polynomial).

The proof of Theorem 1 is given in [9].

Example 11 (continuation of Example 5). To find the cortege of Boolean functions from the polynomial $L A R=2+3 x_{1}+5 x_{2}$, let us express its terms, using (11), as corteges $2=1 \odot 0$, $3 x_{1}=x_{1} \odot x_{1}$, and $5 x_{2}=x_{2} \odot 0 \odot x_{2}$. Hence $L A R=(\overbrace{1 \odot 0}^{2})+(\overbrace{x_{1} \odot x_{1}}^{3 x_{1}})+(\overbrace{x_{2} \odot 0 \odot x_{2}}^{5 x_{2}})$ and

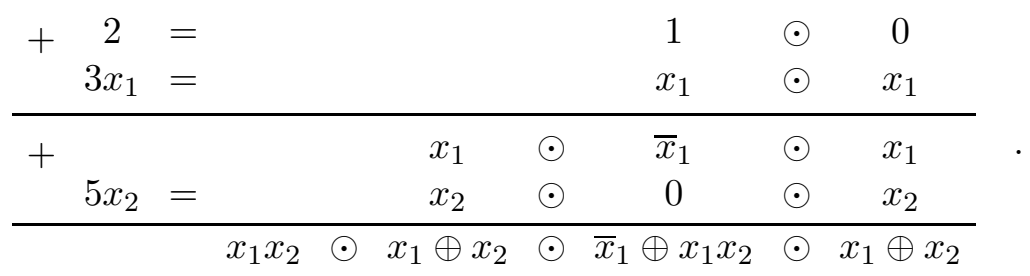

Finally, $L A R=2+3 x_{1}+5 x_{2}=(\overbrace{x_{1} x_{2}}^{f_{4}}) \odot(\overbrace{x_{1} \oplus x_{2}}^{f_{3}}) \odot(\overbrace{x_{1} \oplus x_{1} x_{2}}^{f_{2}}) \odot(\overbrace{x_{1} \oplus x_{2}}^{f_{1}})$.

\section{LINEARIZATION}

As a result of linearization of word-level models, the number of nonzero elements is reduced to the number of variables (2). The LAR is mapped into a linear graph, i.e., a decision diagram. In certain well-known publications, the linear structure of data is determined using different properties of Boolean functions (logical circuits), which must be preliminarily identified. In 1952, Komamiya [17] used a LAR of the type $A_{1}+A_{2}+\ldots+A_{n}=d_{m} 2^{m}+d_{m-1} 2^{m-1}+\ldots+d_{1} 2+d_{0}$ to describe adders, where $A_{i}, d_{i} \in\{0,1\}$. For example, for $n=2$ this relation describes a half-adder, and for $n=3$ a total adder. In this section, we describe the so-called naive or direct approach to linearization, which dominated in investigations for a long time, and the Malyugin approach.

\subsection{Linearization Condition}

A Boolean function $f$ of $n$ variables with $m$ outputs $f_{0}, f_{1}, \ldots, f_{m-1}$ is defined by a truth vector $\left[f^{0} f^{1} \ldots f^{2^{n}-1}\right]$ with integral elements $f^{j}$.

Theorem 2 (linearization condition [10,27]). An m-output Boolean function $f$ of $n$ variables admits representation by LARs if and only if the truth vectors $f^{j}, j \in\left\{0,1, \ldots, 2^{n}-1\right\}$, and $f^{2^{i}}$, $i \in\{0,1, \ldots, n-1\}, j \neq\left\{0,2^{0}, 2^{1}, \ldots, 2^{n-1}\right\}$, satisfy the equality

$$
f^{j}=\sum_{i=0}^{n-1} j_{n-i} f^{2^{i}}+\left(1-\sum_{i=1}^{n} j_{i} f^{0}\right) .
$$


The proof of Theorem 2 is given in [10, 27].

This theorem formulates necessary and sufficient conditions for linearization of arithmetical word-level models of Boolean functions.

Example 12 (continuation of Example 1). The outputs of a half-adder are described by two functions $f_{1}=\left[f_{1}^{0} f_{1}^{1} f_{1}^{2} f_{1}^{3}\right]=[0110]$ (sum) and $f_{2}=\left[f_{2}^{0} f_{2}^{1} f_{2}^{2} f_{2}^{3}\right]=[0001]$ (carry). Let us take a fixed order for the outputs $\left[f_{2} f_{1}\right]$. The linearization condition (12) is satisfied since the equality $2\left(f_{2}^{0}-f_{2}^{1}-f_{2}^{2}+f_{2}^{3}\right)=-f_{1}^{0}+f_{1}^{1}+f_{1}^{2}-f_{1}^{3}$, i.e., $2(0-0-0+1)=-0+1+1-0$ holds for $n=2$. By virtue of the truth vector $\left.\left[\begin{array}{ll}f_{2} & f_{1}\end{array}\right]=\left[\begin{array}{lll}0 & 1 & 1\end{array}\right]\right]^{T}$, we can express the coefficient vector, using, for example, the direct matrix transformation (4): $\mathbf{D}=\left[\begin{array}{llll}0 & 1 & 1 & 0\end{array}\right]$ is the corresponding polynomial $L A R=x_{1}+x_{2}$. If the order of numeration for the outputs of the function is changed to $\left[f_{1} f_{2}\right]$, condition $(12)$ is not satisfied and the half-adder is described by the linear polynomial $2 x_{1}+2 x_{2}-3 x_{1} x_{2}$.

Let us state the constraints in the direct (naive) approach to AR linearization.

Theorem 3. A single-output Boolean function can be uniquely represented by a LAR, which describes a new extended Boolean function with two outputs, one of which is the initial function.

The proof of Theorem 3 is given in the Appendix.

According to Theorem 3, the extended Boolean function contains a garbage function. For example, applying Theorem 3 to the function $f=x_{1} \vee x_{2}=x_{1}+x_{2}-x_{1} x_{2}$, we obtain $L A R=x_{1}+x_{2}+1$ for the cortege $\left(x_{1} \vee x_{2}\right) \odot\left(1 \oplus x_{1} \oplus x_{2}\right)$, where $1 \oplus x_{1} \oplus x_{2}$ is a garbage function. The theorem on the LAR representation of a Boolean function of three variables is proved along similar lines. But serious difficulties are encountered in applying this method to functions of three or more variables.

\subsection{LAR of Primitive Functions}

Malyugin designed an elegant approach to linearization of arithmetical polynomials using cortege algebra [9].

Theorem 4 (LAR of primitive functions [9]). AND, OR, and EXOR functions with $n$ inputs admit representation by $L A R$

$$
\begin{gathered}
n \text {-AND } \bigwedge_{i=1}^{n} x_{i}^{\sigma_{i}}=\boldsymbol{\Xi}_{m}^{m}\left\{2^{j-1}-n+\sum_{i=1}^{n}\left(\sigma_{i}+(-1)^{\sigma_{i}} x_{i}\right)\right\}, \\
n \text {-OR } \bigvee_{i=1}^{n} x_{i}^{\sigma_{i}}=\boldsymbol{\Xi}_{m}^{m}\left\{2^{j-1}-1+\sum_{i=1}^{n}\left(\sigma_{i}+(-1)^{\sigma_{i}} x_{i}\right)\right\}, \\
n-E X O R \quad \bigoplus_{i=1}^{n} x_{i}^{\sigma_{i}}=\boldsymbol{\Xi}_{m}^{1}\left\{\sum_{i=1}^{n}\left(\sigma_{i}+(-1)^{\sigma_{i}} x_{i}\right)\right\},
\end{gathered}
$$

where

$$
j=\left\lceil\log _{2} n\right\rceil+1, \quad x_{i}^{\sigma_{i}}= \begin{cases}x_{i}, & \sigma_{i}=0 \\ \bar{x}_{i}, & \sigma_{i}=1,\end{cases}
$$

and $\lceil a\rceil$ is the integral part of the number $a$.

The proof of Theorem 4 is given in [9]. 
Table 1. Corteges and LAR of primitive elements of the typical CAD IC library

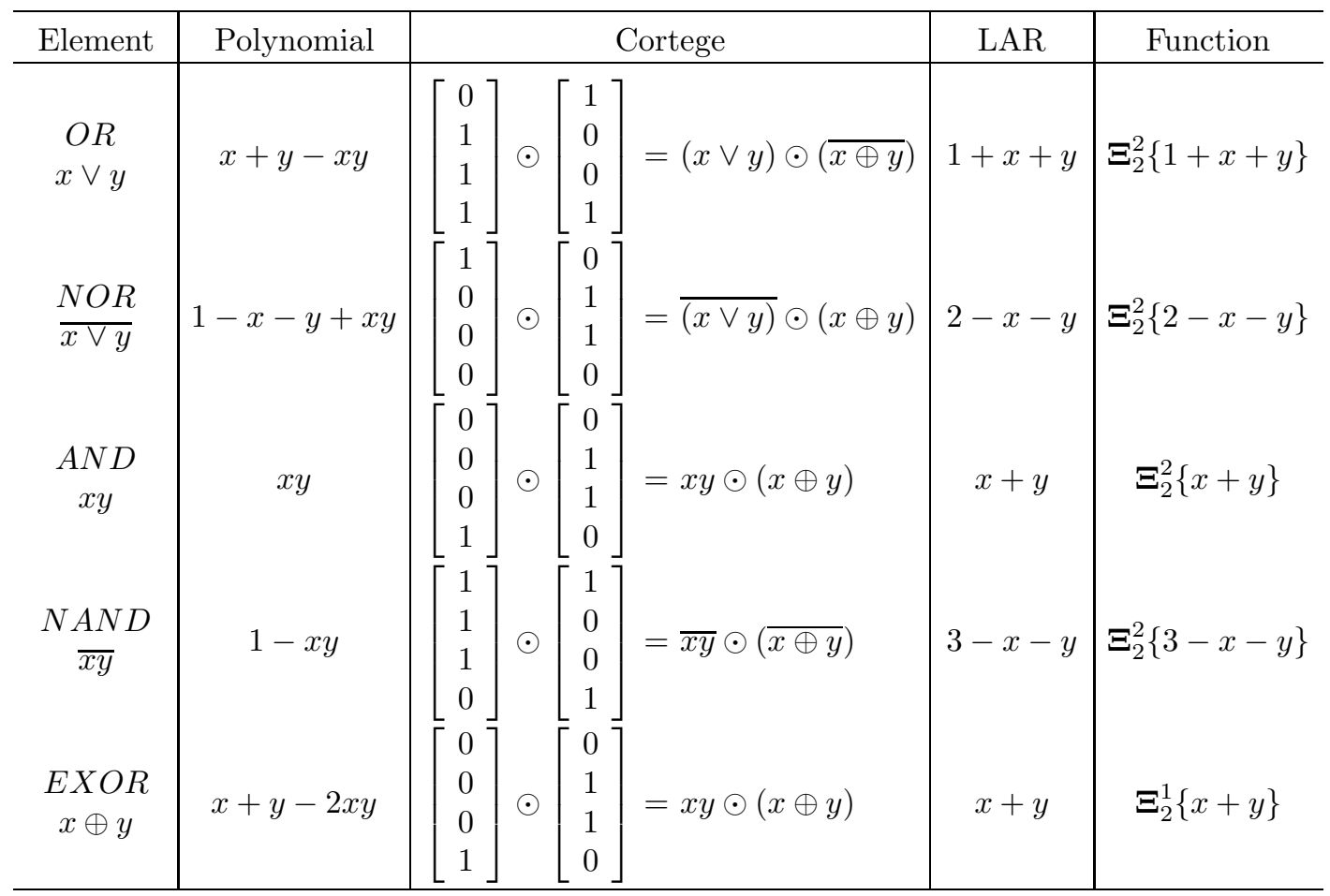

Remark on Theorem 4. ( $i$ ) AND (OR) and EXOR functions correspond to the higher-order bit $(m)$ and lower-order bit of number 1 in word-level format, which is determined by the masking operators $\boldsymbol{\Xi}_{m}^{m}$ and $\boldsymbol{\Xi}_{m}^{1}$, respectively.

(ii) The term $1 \oplus x_{i}^{\sigma_{i}}$ takes either the value $1 \oplus x_{i}=\bar{x}_{i}\left(\sigma_{i}=0\right)$ or the value $x_{i} \oplus \bar{x}_{i}=1\left(\sigma_{i}=1\right)$.

Table 1 illustrates the application of Theorem 4 . In all cases, linearization requires one garbage function to be added. Moreover, the negation operation NOT corresponds to LAR $\boldsymbol{\Xi}_{1}^{1}\{1-x\}$. Using Table 1, we can find LAR of other elements, for example,

$$
\begin{gathered}
\bar{x} \vee y=\boldsymbol{\Xi}_{2}^{2}\{1+(1-x)+y\}=\Xi_{2}^{2}\{2-x+y\}, \\
\bar{x} \oplus y=\boldsymbol{\Xi}_{2}^{1}\{(1-x)+y\}=\Xi_{2}^{1}\{1-x+y\} .
\end{gathered}
$$

LAR for 3-input elements are designed with Theorem 4 along similar lines:

$$
\begin{gathered}
L A R_{O R}=\boldsymbol{\Xi}_{3}^{3}\{3+x+y+z\}, \quad L A R_{N A N D}=\boldsymbol{\Xi}_{3}^{3}\{6-x-y-z\}, \\
L A R_{N O R}=\boldsymbol{\Xi}_{3}^{3}\{4-x-y-z\}, \quad L A R_{E X O R}=\boldsymbol{\Xi}_{3}^{1}\{x+y+z\}, \\
L A R_{A N D}=\boldsymbol{\Xi}_{3}^{3}\{1+x+y+z\}, \quad L A R_{E X N O R}=\boldsymbol{\Xi}_{3}^{1}\{1+x+y+z\} .
\end{gathered}
$$

\subsection{Linear Decision Diagrams}

In this section, we apply the word-level DD technique to manipulate over arithmetical expressions. The linear word-level DD for representing a many-out Boolean function is called the linear decision diagram (LDD), i.e., a word-level decision diagram satisfying linearity conditions.

Definition 3. A linear decision diagram (LDD) is a graph (tree) for representing a linear arithmetical polynomial, whose terminal nodes are put into correspondence with the coefficients of 
the linear arithmetical polynomial, and nodes correspond to variables and realize the arithmetical analog of the Davio expansion $p D_{A}^{8}$ (arithmetic positive Davio expansion) :

$$
f=f_{x_{i}=0}+x_{i}\left(-f_{x_{i}=0}+f_{x_{i}=1}\right) \text { is node, }
$$

where $f_{x_{i}=0}=f\left(x_{1}, \ldots, x_{i-1}, 0, x_{i+1}, \ldots, x_{n}\right)$ and $f_{x_{i}=1}=f\left(x_{1}, \ldots, x_{i-1}, 1, x_{i+1}, \ldots, x_{n}\right)$ are the left and right branches, respectively.

Theorem 5. Every combination circuit ${ }^{9}$ with elements from the standard CAD IC library admits representation by a set of linear decision diagrams.

The proof is implied by the possibility for structuring any logical circuit in the form of a finite number of levels, each of which is described by LARs and direct mapping of a LAR into an LDD (nodes define the operation $p D_{A}$ and hanging vertices represent the LAR coefficients). The order of elements in a level must be fixed so that the finiteness property is preserved. In the general case, LAR and LDD are semi-canonical representations of logical functions.

The complexity of arithmetical models in the form of LAR and LDD is estimated with

Theorem 6. An n-output element of the standard library is represented by an LDD with $n$ nodes and $(n+1)$ hanging vertices.

The proof follows from the recurrent $p D_{A}$ expansion of a LAR of $n$ variables. Consequently, the complexity of representation of a multilevel circuit with $G$ elements is $O(G)$.

Example 13. The linear polynomial $L A R=-3 x_{1}-12 x_{2}+17 x_{3}+20$ is represented by a threevertex $L D D$. Its variables can occur in any order. $L A R_{x_{1}=0}$ and $L A R_{x_{1}=1}$ are

$$
\begin{gathered}
L A R_{x_{1}=0}=-3 \times 0-12 x_{2}+17 x_{3}+20 \text { left branch }, \\
L A R_{x_{1}=1}=-3 \times 1-12 x_{2}+17 x_{3}+20 .
\end{gathered}
$$

According to (14), the term $-f_{x=0}+f_{x=1}$ is equal to the constant $-L A R_{x_{1}=0}+L A R_{x_{1}=1}=-3$. Therefore, the hanging vertex is assigned the value -3 (Fig. 5). Since $L A R_{x_{1}=0}$ is not a constant and $L A R_{x_{1}=0}=-12 x_{2}+17 x_{3}+20$, we use the expansion (left and right branches, respectively)

$$
L A R_{\substack{x_{1}=0 \\
x_{2}=0}}=17 x_{3}+20 \text { and } \quad \begin{array}{r}
L A R_{x_{1}=0} \\
x_{2}=1
\end{array}=-12+17 x_{3}+20 .
$$

Hence the next hanging vertex for $x_{1}=0$ and $x_{2}=0$ takes the value -12 . Decomposition at the next level gives two hanging vertices with values 17 and 20, respectively.

\section{COMPUTATION TECHNIQUE}

\subsection{Weighted Sum of LAR}

The underlying principle of computation is the representation of a finite set of $L A R_{i}$ by the weighted sum $2^{j_{1}} L A R_{0}+2^{j_{2}} L A R_{1}+\ldots+2^{j_{r}} L A R_{r}, j_{1}<j_{2}<\ldots<j_{r}$. The number of bits to represent a LAR is $j=\left\lceil\log _{2}(n)\right\rceil+1$, where $n$ is the number of bits in the representation of the maximal value of the LAR.

\footnotetext{
${ }^{8}$ A distinction is made between the Davio positive $f=f_{0} \oplus x_{1} f_{2}$ and Davio negative $f=\bar{x}_{1} f_{2} \oplus f_{1}$ expansions (Davio's results on AND-EXOR logical expressions are very useful in designing digital devices [28]) and Shannon expansion $f=\bar{x}_{1} f_{0} \oplus x_{1} f_{1}$, where $f_{0}=f\left(0, x_{2}, \ldots, x_{n}\right), f_{1}=f\left(1, x_{2}, \ldots, x_{n}\right), f_{2}=f_{0} \oplus f_{1}$.

${ }^{9}$ In the general case, every memory circuit can be represented by LAR and LDD.
} 


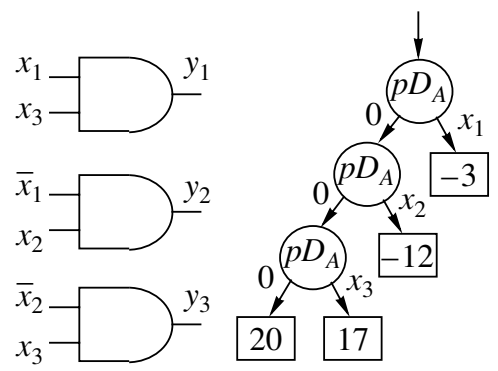

Fig. 5. Levels of a three-AND element circuit corresponding to $L A R=-3 x_{1}-12 x_{2}+17 x_{3}+20$ and LDD.
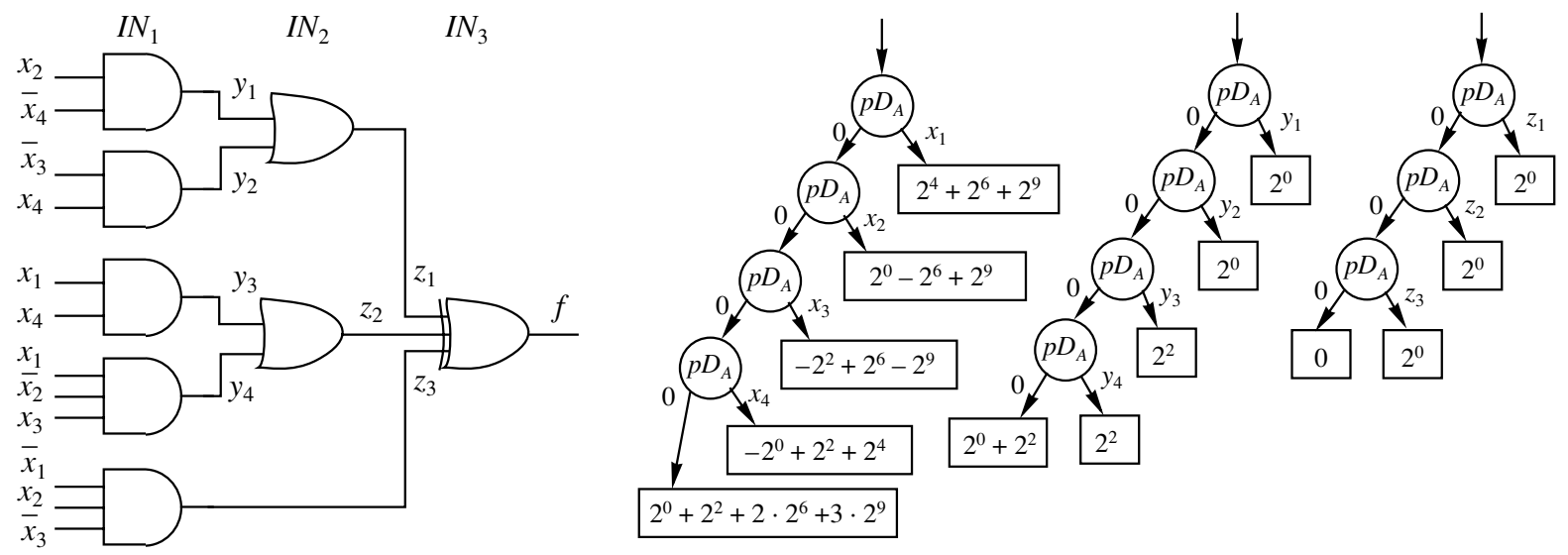

Fig. 6. An AND/OR/EXOR circuit (left) and its LDD system (right).

Example 14. Let us consider the levels of a three-element circuit (Fig. 5). The first element represents a LAR of weight $2^{0}=1$ and $j_{1}=\left\lceil\log _{2}(2)\right\rceil+1=2$ bits are required for its representation. The second and third LARs have weights $2^{2}$ and $2^{4}$ and require $j_{2}=2$ and $j_{1}+j_{2}=4$ bits, respectively. Consequently, the circuit level in Fig. 5 is described by the LAR

$$
\begin{gathered}
L A R=2^{0}\left(x_{1}+x_{2}\right)+2^{2}\left(\bar{x}_{1}+x_{2}\right)+2^{4}\left(\bar{x}_{2}+x_{3}\right) \\
=2^{0}\left(x_{1}+x_{2}\right)+2^{2}\left(1-x_{1}+x_{2}\right)+2^{4}\left(1-x_{2}+x_{3}\right)=-3 x_{1}-12 x_{2}+17 x_{3}+20 .
\end{gathered}
$$

The resultant LAR is the linear model for the $l$ th level $I N_{l}$ of the circuit with $n$ inputs and $r$ outputs. Representation of the $i$ th LAR requires a memory $J_{i}=j_{1}+\ldots+j_{i-1}+j_{i}=J_{i-1}+j_{i}$, where $i \in\{1,2, \ldots, r\}$ and $J_{0}=0$. Computation with this model is reduced to computing the weighted sum $L A R_{i}, i \in\{1, \ldots, r\}$ :

$$
L A R=I N_{l}=\sum_{i=1}^{r}\left(2^{J_{i}} L A R_{i}\right),
$$

where $j_{i}=\left\lceil\log _{2} n\right\rceil+1$ is the number of bits required to represent $L A R_{i}$.

\subsection{Representation of Multilevel Circuits by a System of LDD}

Using Theorem 5 and Example 13, let us describe a method for designing linear models of LAR and LDD for any logical circuit consisting of element of the standard CAD IC library. 
Example 15. An AND/OR/EXOR circuit (Fig. 6) is given. Using the LAR model of elements in Table 1, let us construct an LDD model. The LAR of the first circuit level is

$$
\begin{array}{lll}
L A R_{1}= & 2^{0}\left(1+x_{2}-x_{4}\right) & \Rightarrow \text { element } 1 \\
+ & 2^{2}\left(1+x_{4}-x_{3}\right) & \Rightarrow \text { element } 2 \\
+ & 2^{4}\left(x_{1}+x_{4}\right) & \Rightarrow \text { element } 3 \\
+ & 2^{6}\left(2+x_{1}-x_{2}+x_{3}\right) & \Rightarrow \text { element } 4 \\
+ & 2^{9}\left(3-x_{1}+x_{2}-x_{3}\right) & \Rightarrow \text { element } 5
\end{array}
$$

Weights required for describing the second circuit level and the corresponding LARs are $y_{1}=$ $\boldsymbol{\Xi}^{2}\left\{L A R_{1}\right\}, y_{2}=\boldsymbol{\Xi}^{4}\left\{L A R_{1}\right\}, y_{3}=\boldsymbol{\Xi}^{6}\left\{L A R_{1}\right\}$, and $y_{4}=\boldsymbol{\Xi}^{9}\left\{L A R_{1}\right\}, L A R_{2}=2^{0}\left(1+y_{1}+y_{2}\right)+$ $2^{2}\left(1+y_{3}+y_{4}\right)$. Similarly, LAR of the third circuit level are $z_{1}=\boldsymbol{\Xi}^{2}\left\{L A R_{2}\right\}, z_{2}=\boldsymbol{\Xi}^{4}\left\{L A R_{2}\right\}$, $z_{3}=\boldsymbol{\Xi}^{12}\left\{L A R_{1}\right\}$ and $L A R_{2}=z_{1}+z_{2}+z_{3}$. Finally, the circuit output is $f=\boldsymbol{\Xi}^{1}\left\{L A R_{3}\right\}$. The LDD model is shown in Fig. 6.

Even in this simple 8-element circuit, coefficients take values as high as $2^{12}$. Industrial circuits contain millions of elements. This is the motivation for developing new approaches to designing linear models.

\section{LARGE WEIGHT COEFFICIENTS}

Arithmetical models cannot be used in CAD IC due to the large values of coefficients even in circuits with 10-20 inputs. The well-known approaches based on modular arithmetic [30] only partly solve this problem, which we now we solve using the properties of the coefficients of LARs.

\subsection{Properties of Coefficients}

Hypothesis. Since every logical circuit is designed from a bounded number of standard elements with known LAR (Table 1), we may successfully apply the properties of linear models based on simple linear models of primitive elements.

Theorem 7. The lth level $I N_{l}$ of a logical circuit with $n$ inputs and $r$ elements admits representation by a LAR

$$
I N_{l}=W_{0}+W_{x_{1}} x_{1}+\ldots+W_{x_{n}} x_{n}
$$

where the weights of hanging vertices $W_{0}$ and $W_{x_{i}}, i=1, \ldots, n$, are

$$
W_{0}=a_{0,1} 2^{J_{1}}+\ldots+a_{0, r} 2^{J_{r}}, \text { and } W_{x_{i}}=a_{i, 1} 2^{J_{1}}+\ldots+a_{i, r} 2^{J_{r}},
$$

$a_{i, t} \in\{0,+1,-1\}$ and $a_{0, t}$ are positive integers, $J_{1}=j_{0}=0, J_{t}=j_{0}+\ldots+j_{t-1}, j_{t}=\left\lceil\log _{2} n_{t}\right\rceil+1$, and $n_{t}$ is the number of inputs of the function obtained by extending $y_{t}$ for deriving $L A R\left(y_{t}\right)$.

The proof of Theorem 7 is given in the Appendix.

Corollary 1. The parameters $J_{i}$, except for $J_{0}=0$, are defined by the difference $\mathrm{M}=J_{i}-J_{i-1}$ since the coefficients of hanging vertices are the sum of weights $2^{J_{i}}$, where $J_{i}$ is the sum of $j_{l}(l<i)$.

Theorem 7 and Corollary 1 yield a coding algorithm based on the substitution of the values of hanging vertices by the respective codes. Let us describe this algorithm with an example (for details, see $[12,31])$. Consider the LDD in Fig. 5. The first hanging vertex is assigned the value -3 . Since the weight of the LAR of the first element is $2^{0}\left(J_{1}=0\right)$ and $x_{1}$ is contained without negation 


\begin{tabular}{lcccccccccc|} 
Index & 9 & 8 & 7 & 6 & 5 & 4 & 3 & 2 & 1 \\
Weight & $2^{24}$ & $2^{21}$ & $2^{18}$ & $2^{15}$ & $2^{12}$ & $2^{9}$ & $2^{6}$ & $2^{3}$ & $2^{0}$ \\
\cline { 2 - 11 } Code & 01 & 01 & 00 & 00 & 11 & 11 & 11 & 01 & 00 \\
\hline
\end{tabular}

Fig. 7. Coding of the hanging vertex in LDD (Example 16).

$\left(b_{1}=1\right)$. The weight of LAR for the second element is $2^{2}\left(J_{2}=2\right)$ and $x_{1}$ is contained with negation $\left(b_{1}=1\right)$. Consequently,

$$
W_{x_{1}}=b_{1} 2^{J_{1}}+b_{2} 2^{J_{2}}=12^{0}-12^{2}=-3 .
$$

Relation (18) shows that the number -3 is uniquely represented by four attributes $b_{1}, J_{1}, b_{2}$, and $J_{2}$. Obviously, $b_{i}$ and $J_{i}$ require far less memory than the weights of hanging vertices for storage. Indeed, since $b_{i}$ can take only three values $b_{i} \in\{0,1,-1\}$, two bits are sufficient for its code, for example, $0 \Rightarrow 00,1 \Rightarrow 01$, and $-1 \Rightarrow 11$.

According to Theorem 7 and Corollary 1, the coefficients of hanging vertices are not arbitrary integers. Consequently, continuing the example, we find that it suffices to store only $J_{1}=0$ and $J_{2}-J_{1}=2$. Therefore, for the first hanging vertex, we must store the parameters $b_{1}=01, J_{1}=000$, $b_{2}=11$, and $J_{2}-J_{1}=j_{2}=010$.

Now we consider the second hanging vertex. Its weight is $W_{x_{2}}=b_{1} 2^{J_{1}}+b_{2} 2^{J_{2}}=1 \times 2^{2}-1 \times 2^{4}=$ -12 , which can be represented by four parameters $b_{1}=01, J_{1}=010, b_{2}=11$, and $j_{2}=010$. Similarly, the weight of the third hanging vertex is $W_{x_{3}}=b_{1} 2^{J_{1}}+b_{2} 2^{J_{2}}=1 \times 2^{0}+1 \times 2^{4}=20$. But it is sufficient to store $b_{1}=01, J_{1}=000, b_{2}=01$, and $J_{2}-J_{1}=100$. The parameters of the free hanging vertex are $a_{1}=01, J_{1}=000, b_{2}=01$, and $J_{2}-J_{1}=010$, which correspond to the weight $W_{0}=a_{1} 2^{J_{1}}+a_{2} 2^{J_{2}}=1 \times 2^{0}+1 \times 2^{2}$.

Example 16. The code of the value $0+2^{3}-2^{6}-2^{9}-2^{12}+0+0+2^{21}+2^{24}$ of the hanging vertex is shown in Fig. 7. The coefficient of the linear model attains the value $2^{24}$ and is coded by 18 bits.

\section{EXPERIMENTAL RESULTS}

Results of experiments conducted with large-dimensional test circuits are reported in [12]. Every circuit (including, circuits with multivalued gates) is shown to be representable by a finite set of LAR and LDD. A fragment of experiments is listed in Table 2, where TEST, \#IN, \#OUT, and \#G denote the name, number of inputs, outputs, and gates of the circuit ${ }^{10}$, respectively. The columns EDIF,

Table 2. Memories for LDD, EDIF, ISCAS models

\begin{tabular}{lrrr|rr|rr}
\hline TEST & \#IN & \#OUT & \#G & EDIF & LDD & ISCAS & LDD \\
\hline C1355 & 41 & 32 & 546 & 273018 & 16133 & 59573 & 16850 \\
C1908 & 33 & 25 & 880 & 230507 & 13098 & 78574 & 22491 \\
C2670 & 233 & 108 & 1193 & 400707 & 32395 & 110025 & 36305 \\
C3540 & 50 & 24 & 1669 & 503457 & 34744 & 145359 & 54857 \\
C5315 & 178 & 163 & 2307 & 903899 & 88965 & 220596 & 107392 \\
C6288 & 32 & 32 & 2416 & 1387230 & 71476 & 255406 & 68572 \\
C7552 & 207 & 109 & 3512 & 1236066 & 146057 & 311939 & 271970 \\
\hline
\end{tabular}

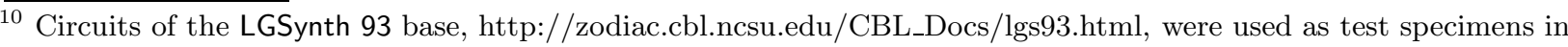
experiments.
} 
ISCAS, and LDD show memory (in bytes) for the traditional representation of a circuit in EDIF, ISCAS, and LDD formats. Clearly, LDD models require 9-25 times less memory than traditional models. For example, the combination circuit C7552 with 207 inputs, 109 outputs, and 3512 gates requires 1236066 and 162222 bytes for storage in EDIF and ISCAS formats, respectively, i.e., 22 and 10 times less memory for storage than in LDD format. Verification of linear circuit models is still an unresolved problem.

\section{ARITHMETICAL LOGIC IN NANOTECHNOLOGY}

The deterministic models described in this paper may not find direct utilization in future technologies due to the stochastic behavior of nanostructures [32]. This stimulates the study of probabilistic and information models for computation. Arithmetical logic is the boundary case of the so-called probabilistic logic. Merekin [33] was probably the first to prove that the reliability function of the switching circuit is the generalization to arithmetical description of the circuit. Malyugin used this property to study the reliability of switching circuits with arithmetical polynomials [34].

Example 17. Let signals $x_{1}$ and $x_{2}$ appear at the input of an OR gate independently with probabilities $p_{1}=p\left(x_{1}\right)$ and $p_{2}=p\left(x_{2}\right)$, respectively. The output $y$ of the OR gate is either $x_{1}$ or $x_{2}$. Let $p_{1}=0.8$ and $p_{2}=0.9$. Then the correct output must be expected with probability $p=1-\left(1-p_{1}\right)\left(1-p_{2}\right)=p_{1}+p_{2}-p_{1} p_{2}=0.8+0.9-0.8 \times 0.9=0.98$.

Table 3. Deterministic and probabilistic models of typical gates

\begin{tabular}{c|c|c|c}
\hline Gate & Conjunctor & Disjunctor & Mod 2 adder \\
\hline \multicolumn{4}{|c}{ Deterministic model } \\
Function & $y=x_{1} x_{2}$ & $y=x_{1} \vee x_{2}$ & $y=x_{1} \oplus x_{2}$ \\
Polynomial & $y=x_{1} x_{2}$ & $y=x_{1}+x_{2}-x_{1} x_{2}$ & $y=x_{1}+x_{2}-2 x_{1} x_{2}$ \\
LAR & $\boldsymbol{\Xi}_{2}^{2}\left\{x_{1}+x_{2}\right\}$ & $\boldsymbol{\Xi}_{2}^{2}\left\{1+x_{1}+x_{2}\right\}$ & $\mathbf{\Xi}_{2}^{1}\left\{x_{1}+x_{2}\right\}$ \\
\multicolumn{4}{|c|}{ Probabilistic model } \\
Model & $p=p_{1} p_{2}$ & $p=p_{1}+p_{2}-p_{1} p_{2}$ & $p=p_{1}+p_{2}-2 p_{1} p_{2}$ \\
\hline
\end{tabular}

Taking $p_{1}=p_{2}=1$, let us transform the probabilistic model into a deterministic model. Obviously, the output of the OR element is $y=x_{1}+x_{2}-x_{1} x_{2}$, which is the arithmetical description of the OR element. Table 3 shows the probabilistic characteristics of AND, OR, and EXOR gates.

\section{CONCLUSIONS}

Malyugin's theorems jointly with the latest results in arithmetical logic and modern CAD IC tools are effective in linearizing arithmetical forms of representation of not only Boolean functions, but also multivalued logical functions, as demonstrated in [12]. Possibly, these results may be useful in future technologies and other problems, viz., in artificial intelligence systems.

\section{ACKNOWLEDGMENTS}

I express my indebtedness to S. Yanushkevich, G. Duek (Canada), P. Dziurzanski, T. Lub (Poland), Sasao (Japan), M. Karkovsky (USA), B. Falkowski (Singapore), O. Fin'ko (Russia), and R. Drechsler (Germany) for their valuable discussion and assistance at different stages of preparation of the paper. I also express my thanks to the referees for their remarks. 
Proof of Theorem 3. Each of $16^{2}$ possible pairs of 16 Boolean functions of two variables uniquely corresponds to an arithmetical expression also containing LAR (2). Indeed, two functions are defined by four values $f=\left(f_{0}, f_{1}, f_{2}, f_{3}\right)$ and $f^{\prime}=\left(f_{0}^{\prime}, f_{1}^{\prime}, f_{2}^{\prime}, f_{3}^{\prime}\right)$ and represent 256 possible two-output Boolean functions $f$ and $f^{\prime}$ or an integer function with values $\left(2 f_{0}+f_{0}^{\prime}, 2 f_{1}+f_{1}^{\prime}, 2 f_{2}+\right.$ $\left.f_{2}^{\prime}, 2 f_{3}+f_{3}^{\prime}\right)$. These functions correspond to the arithmetical expression $2^{1} f+2^{0} f^{\prime}$ and satisfy condition (2) if

$$
2 f_{3}+f_{3}^{\prime}=-\left(2 f_{0}+f_{0}^{\prime}\right)+\left(2 f_{1}+f_{1}^{\prime}\right)+\left(2 f_{2}+f_{2}^{\prime}\right) .
$$

Expression (19) holds only for 44 two-outputs functions out of 256 functions. But each of 16 subfunctions occurs at least once at the first and second places. Consequently, any one-output function of two variables can be represented by LAR via extension, i.e., addition of a function in word level format such that the result is a LAR.

Proof of Theorem 7. Let the output of the first element belonging to the level be described by the expression $\operatorname{LAR}\left(y_{1}\right)=2^{1} f_{2}+2^{0} f_{1}$. Since $A R\left(f_{1}\right)=a_{0.1}+a_{1.1} x_{2}+a_{2.1} x_{1}+a_{3.1} x_{1} x_{2}$ and $A R\left(f_{2}\right)=a_{0.2}+a_{1.2} x_{2}+a_{2.2} x_{1}+a_{3.2} x_{1} x_{2}$, we obtain

$$
\begin{gathered}
\operatorname{LAR}\left(y_{1}\right)=2^{1}\left(a_{0.2}+a_{1.2} x_{2}+a_{2.2} x_{1}+a_{3.2} x_{1} x_{2}\right)+2^{0}\left(a_{0.1}+a_{1.1} x_{2}+a_{2.1} x_{1}+a_{3.1} x_{1} x_{2}\right) \\
=\left(2^{1} a_{0.2}+2^{0} a_{0.1}\right)+\left(2^{1} a_{1.2}+2^{0} a_{1.1}\right) x_{2}+\left(2^{1} a_{2.2}+2^{0} a_{2.1}\right) x_{1}+\left(2^{1} a_{3.2}+2^{0} a_{3.1}\right) x_{1} x_{2} .
\end{gathered}
$$

Discarding nonlinear terms (all components are linear), we obtain $\operatorname{LAR}\left(y_{1}\right)=\left(2^{1} a_{0.2}+2^{0} a_{0.1}\right)+$ $\left(2^{1} a_{1.2}+2^{0} a_{1.1}\right) x_{2}+\left(2^{1} a_{2.2}+2^{0} a_{2.1}\right) x_{1}$. Let $W_{0}=2^{1} a_{0.2}+2^{0} a_{0.1}, W_{x_{1}}=2^{1} a_{1.2}+2^{0} a_{1.1}$, and $W_{x_{2}}=2^{1} a_{2.2}+2^{0} a_{2.1}$. Then $\operatorname{LAR}\left(y_{1}\right)=W_{0}+W_{x_{1}} x_{1}+W_{x_{2}} x_{2}$ (weights are determined with lowerorder digits of the values of the function in LAR). Applying the same approach, we obtain LARs of other elements.

\section{REFERENCES}

1. Bryant, R. and Chen, Y., Verification of Arithmetic Functions Using Binary Moment Diagrams, Proc. Design Automat. Conf., 1995, pp. 535-541.

2. Drechsler, R., Formal Verification of Circuits, Boston: Kluwer, 2000.

3. Lai, Y., Pedram, M., and Vrudhula, S., EVBDD-based Algorithms for Integer Linear Programming, Spectral Transformation, and Function Decomposition, IEEE Trans. CAD Integrated Circuits Syst., 1994, vol. 13, no. 8, pp. 959-975.

4. Minato, S., Binary Decision Diagrams and Applications for VLSI CAD, Boston: Kluwer, 1996.

5. Aiken, H.H., The Annals of the Computation Laboratory of Harvard University, Synthesis Electron. Comput. Control Circuits, Cambridge: Harvard University, 1951, vol. XXVII.

6. Huang, C.-Y. and Cheng, K.-T., Using Word-Level ATPG and Modular Arithmetic Constraint-Solving Techniques for Assertion Property Checking, IEEE Trans. CAD Integrated Circuits Syst., 2001, vol. 20, no. 3, pp. 381-391.

7. Rahardia, S. and Falkowski, B.J., Fast Linearly Independent Arithmetic Expansion, IEEE Trans. Comput., 1999, vol. 48, no. 9, pp. 991-999.

8. Malyugin, V.D., Realization of Boolean Functions by Arithmetical Polynomials, Avtom. Telemekh., 1982, no. 4, pp. 84-93.

9. Malyugin, V.D., Realization of a Cortege of Boolean Functions by Linear Arithmetical Polynomials, Avtom. Telemekh., 1984, no. 2, pp. 114-121.

10. Antonenko, V.M., Ivanov, A.A., and Shmerko, V.P., Linear Arithmetical Forms of k-valued Logical Functions and Their Realization by Systolic Arrays, Avtom. Telemekh., 1995, no. 3, pp. 139-155.

11. Shmerko, V.P., Synthesis of Arithmetical Forms of Boolean Functions through Fourier Transformation, Avtom. Telemekh., 1989, no. 5, pp. 134-142. 
12. Dziurzanski, P., Malyugin, V., Shmerko, V., and Yanushkevich, S., Linear Models of Circuits of Multivalued Elements, Avtom. Telemekh., 2002, no. 6, pp. 99-119.

13. Falkowski, B.J., A Note on the Polynomial Form of Boolean Functions and Related Topics, IEEE Trans. Comput., 1999, vol. 48, no. 8, pp. 860-863.

14. Jain, J., Bitner, J., Fussell, D.S., and Abraham, J.A., Probabilistic Verification of Boolean Functions, in: Formal Methods in Syst. Design, Boston: Kluwer Academic, 1992, vol. 1, pp. 61-115.

15. Thornton, M.A., Dreschler, R., and Miller, D.M., Spectral Techniques in VLSI CAD, London: Kluwer Academic, 2002.

16. Nechiporuk, E.I., Design of Circuits via Linear Transformations of Variables, Dokl. Akad. Nauk SSSR, 1958, vol. 123, no. 4, pp. 610-612.

17. Stankovic, R.S. and Sasao, T., Discussion on the History of Research in Arithmetic and Reed-Muller Expressions, IEEE Trans. CAD of Integrated Circuits and Syst., 2001, vol. 20, no. 9, pp. 1177-1179.

18. Eris, E. and Muzio, J.C., Spectral Testing of Circuit Realisations Based on Linearizations, IEE Proc., Part. E, 1986, vol. 133, no. 2, pp. 73-78.

19. Karpovsky, M.G. and Moskalev, E.S., Utilization of Autocorrelation Characteristics for the Realization of Systems of Logical Functions, Avtom. Telemekh., 1970.

20. Kolpakov, A.V. and Latypov, R.Kh., Approximate Algorithm for Minimization of Binary Decision Diagrams based on Linear Transformations of Variables, Avtom. Telemekh., 2004, no. 6.

21. Stankovic, R.S. and Astola, J.T., Some Remarks on Linear Transform of Variables in Adders, Proc. 5th Int. Workshop on Applications of the Reed-Muller Expansions in Circuit Design, Mississipi State Univ., 2001, pp. 294-302.

22. Heidtmann, K.D., Arithmetic Spectrum Applied to Fault Detection for Combinational Networks, IEEE Trans. Comput., 1991, vol. 40, no. 3, pp. 320-324.

23. Papaioannou, S.G., Optimal Test Generation in Combinational Networks by Pseudo-Boolean Programming, IEEE Trans. Comput., 1977, vol. 26, pp. 553-560.

24. Yanushkevich, S., Arithmetical Canonical Expansions of Boolean and MVL Functions as Generalized Reed-Muller Series, Proc. IFIP WG 10.5 Workshop on Appl. Reed-Muller Expansions in Circuit Design, Japan, 1995, pp. 300-307.

25. Yanushkevich, S., Systolic Algorithm for Designing Arithmetical Polynomial Forms of $k$-valued Functions of Logical Algebra, Avtom. Telemekh., 1994, no. 12, pp. 128-142.

26. Akers, S.B., On a Theory of Boolean Functions, SIAM, 1959, vol. 7, no. 4, pp. 487-498.

27. Artyukhov, V.L., Kondrat'ev, V.N., and Shalyto, A.A., Realization of Boolean Functions by Arithmetical Polynomials, Avtom. Telemekh., 1988, no. 4, pp. 138-147.

28. Davio, M.J., Deschamps, P., and Thayse, A., Discrete and Switching Functions, New York: McGrawHill, 1978.

29. Malyugin, V.D., Parallel'nye logicheskie vychisleniya posredstvom arifmeticheskikh polinomov (Parallel Logical Computation through Arithmetical Polynomials), Moscow: Nauka, 1997.

30. Fin'ko, O.A., Realization of Large-Dimensional Systems of Boolean Functions by Modular Arithmetical Methods, Avtom. Telemekh., 2004, no. 6.

31. Yanushkevich, S., Dziurzanski, P., and Shmerko, V., Word-Level Models for Efficient Computation of Multiple-Valued Functions: LAR, IEEE 32th Int. Symp. Multiple-Valued Logic, Boston, 2002, pp. 202-208.

32. Yamada, T., Kinoshita, Y., Kasai, S., Hasegawa, H., and Amemiya, Y., Quantum-Dot Logic Circuits Based on Shared Binary Decision Diagram, Jpn. J. Appl. Phys., 2001, vol. 40, part 1, no. 7, pp. 4485-4488.

33. Merekin, Yu.V., Arithmetical Forms of Expression for Boolean Functions and Their Application in Circuit Reliability Computations, Vychisl. Syst., 1963, vol. 7.

34. Malyugin, V.D., Reliability of Switching Circuits, Avtom. Telemekh., 1964, no. 9, pp. 1375-1383.

This paper was recommended for publication by P.P. Parkhomenko, a member of the Editorial Board 